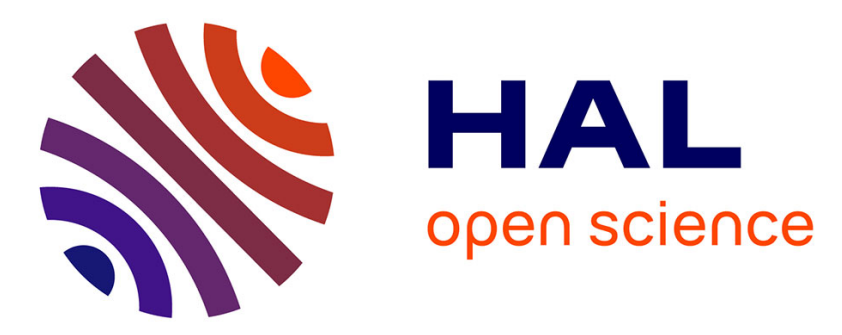

\title{
De la traversée clandestine à la visibilité urbaine: réfugiés et demandeurs d'asile africains dans les villes israéliennes
}

Lisa Anteby-Yemini

\section{- To cite this version:}

Lisa Anteby-Yemini. De la traversée clandestine à la visibilité urbaine: réfugiés et demandeurs d'asile africains dans les villes israéliennes. Méditerranée: revue géographique des pays méditerranéens, 2009, Migrations et territoires de la mobilité en Méditerranée, 113, pp.14-24. 10.4000/mediterranee.3610 . hal-01409953

\section{HAL Id: hal-01409953 \\ https://hal.science/hal-01409953}

Submitted on 6 Dec 2016

HAL is a multi-disciplinary open access archive for the deposit and dissemination of scientific research documents, whether they are published or not. The documents may come from teaching and research institutions in France or abroad, or from public or private research centers.
L'archive ouverte pluridisciplinaire HAL, est destinée au dépôt et à la diffusion de documents scientifiques de niveau recherche, publiés ou non, émanant des établissements d'enseignement et de recherche français ou étrangers, des laboratoires publics ou privés. 


\section{Méditerranée}

Revue géographique des pays méditerranéens / Journal of Mediterranean geography

\section{De la traversée clandestine à la visibilité urbaine:} réfugiés et demandeurs d'asile africains dans les villes israéliennes

From clandestine crossings to urban visibility: African refugees and asylum seekers in Israeli towns

\section{Lisa Anteby-Yemini}

\section{revues.org}

Édition électronique

URL : http://mediterranee.revues.org/3610

DOI : 10.4000/mediterranee.3610

ISSN : $1760-8538$

\section{Éditeur}

Presses Universitaires de Provence

Édition imprimée

Date de publication : 31 décembre 2009

Pagination : 14-24

ISBN : 978-2-85399-745-4

ISSN : 0025-8296

Référence électronique

Lisa Anteby-Yemini, « De la traversée clandestine à la visibilité urbaine: réfugiés et demandeurs d'asile africains dans les villes israéliennes », Méditerranée [En ligne], 113| 2009, mis en ligne le 31 décembre 2011, consulté le 01 octobre 2016. URL : http://mediterranee.revues.org/3610 ; DOI : 10.4000/ mediterranee. 3610

Ce document est un fac-similé de l'édition imprimée. 


\section{De la traversée clandestine à la visibilité urbaine: réfugiés et demandeurs d'asile africains dans les villes israéliennes}

From clandestine crossings to urban visibility: African refugees and asylum seekers in Israeli towns

Lisa ANTEBY-YEMINI

anthropologue

chargée de recherches CNRS

IDEMEC

anteby@mmsh.univ-aix.fr

\begin{abstract}
Cet article traite des réfugiés et demandeurs d'asile africains, qui traversent clandestinement la frontière d’Égypte vers Israël de façon massive depuis 2005, dans une perspective à la fois diachronique, les situant par rapport aux migrations non-juives vers Israël (en particulier les précédents migrants de travail africains), et dans une dimension synchronique, examinant leurs spécificités face aux autres migrants actuellement en Israël. Si les demandeurs d'asile s'insèrent dans l'espace urbain israélien et dans les communautés existantes de migrants, reproduisant des modèles de territorialisation, d'insertion économique et de structuration communautaire similaires, ils présentent aussi de nouvelles formes de visibilité dans l'espace public et dans la société civile et utilisent une rhétorique de victimes en miroir avec le passé juif, qui reconfigurent le paysage migratoire d'Israël.
\end{abstract}

Mots clés: demandeurs d’asile, réfugiés, sub-sahariens, Israël, espace urbain

Depuis les années 1990, Israël, qui n’avait jamais accueilli de migrations non-juives, connaît un nombre croissant de travailleurs étrangers, certains détenteurs de permis de travail, principalement d'Europe de l'Est et d'Asie, et d’autres, irréguliers, arrivant généralement comme touristes d'Afrique et d'Amérique du Sud. Par le biais d'agences de recrutement ou sur des visas touristiques, ces migrants arrivent quasi-exclusivement par l'aéroport Ben-Gurion de Tel-Aviv. En 2003 des expulsions massives font pratiquement disparaître les communautés africaines (estimées entre 10000 et 14000 personnes selon Sabar, 2006) et latino-américaines.

Début 2000, se développe un nouveau flux migratoire, essentiellement en provenance d'Afrique (Érythrée, Soudan, Côte-d'Ivoire), caractérisé par une majorité de demandeurs d'asile, franchissant clandestinement la frontière israéloégyptienne qui devient en l'espace de quelques années le point majeur d'entrée illégale vers le territoire israélien (Anteby-Yemini, 2008). La plupart obtiennent une protection temporaire, quelques-uns reçoivent le statut de réfugié, et d'autres sont déboutés, devenant alors irréguliers s'ils ne quittent pas le pays.

Cet article se propose d'examiner comment ces demandeurs d'asile s'insèrent dans l'espace urbain israélien et dans les communautés existantes de migrants de travail mais aussi
This article deals with African refugees and asylum-seekers who have been crossing the border from Egypt to Israel clandestinely in vast numbers since 2005. It is going to look at this situation from both a diachronic perspective, which situates them in relation to non-Jewish migrants to Israel (in particular the former African working migrants) and a synchronic perspective, which examines their specificities in relation to other migrants in Israel today. If the asylum-seekers are integrating into the Israeli urban landscape as well as into the existing communities of migrants, reproducing similar models of territorialization, economic integration and communal organization, they are also demonstrating new forms of visibility in the public space and in civil society and are using a discourse as victims mirroring the Jewish past, which is reconfiguring the Israeli migratory landscape.

Keywords: asylum-seekers, refugees, Africans, Israel, urban space

comment ils s'en différencient par une visibilité et des revendications spécifiques en tant que « réfugiés ». Ces questions seront analysées par le prisme de deux « usages du passé »: l'usage des infrastructures, des espaces symboliques et des « mémoires » laissées par les migrations africaines de travail précédentes (quasi-inexistantes aujourd'hui) ainsi que par l'usage d'un discours et une mobilisation articulés autour du parallèle entre passé juif et persécutions africaines contemporaines. Ces deux angles d'approche tenteront ainsi d'illustrer la question du "passé du présent » que ce soit dans le temps court de la « minceur » historique des migrations africaines non-juives en Israël ou dans le temps long de l'identité juive du pays d'accueil.

Nous commencerons par examiner en quoi les demandeurs d'asile constituent une nouvelle catégorie dans l'espace migratoire d'Israël avec des statuts juridiques inédits, face aux migrants de travail, et pourquoi la nouveauté et l'ampleur de cette migration entraînent encore à l'heure actuelle une politique d'accueil incohérente. En effet, à la différence des migrations économiques, cette migration forcée pose un défi à la fois sécuritaire (par l'entrée illégale par une frontière terrestre) et humanitaire (par l'arrivée d'individus demandant une protection internationale et un asile territorial) résultant en détentions prolongées mais aussi en octroi de permis de séjour temporaires.

I Cet article est en partie tiré de deux communications: «Les demandeurs d’asile africains en Israël: une nouvelle catégorie dans le paysage migratoire » $8^{\text {th }}$ Mediterranean Social and Political Research Meeting, European University Institute, 21-25 mars 2007, Monticatini, Italie et « Israël: un nouvel espace de transit pour les migrations africaines », Journée IDEMEC/IREMAM/LAMES/TELEMME Migrations et territoires de la mobilité dans l'espace méditerranéen: «le présent du passé », 23 nov. 2007, MMSH, Aix-en-Provence. 
Dans un deuxième temps, nous verrons comment ces demandeurs d'asile deviennent des résidents dans les centres urbains israéliens (notamment à Tel-Aviv) tout en s’intégrant spatialement, culturellement, politiquement et religieusement dans les communautés étrangères existantes, démontrant une continuité avec ces migrations précédentes dans ses pratiques de territorialisation dans la ville. En revanche, on observe une rupture avec les migrations de travail, particulièrement dans les discours, les mobilisations (dans la société civile) et les revendications de ces nouveaux arrivants en tant que « réfugiés » qui font usage du passé juif et de l'expérience commune d'exil et de génocide des Africains et des Israéliens pour justifier leur demande de protection ${ }^{2}$.

\section{I - Une nouvelle catégorie dans le paysage migratoire israelien}

La migration de demandeurs d'asile vers Israël est une nouveauté du point de vue de ses caractéristiques, de par l'entrée clandestine par la frontière terrestre avec l'Égypte, le dépôt de demande d'asile par la quasi-totalité des migrants, les différents statuts, visas et permis octroyés, les situations inédites d'irrégularité de certains (déboutés), le traitement - parfois critiqué - de ces migrants par les autorités israéliennes, et de par la politique simultanée de répression et d'accueil qui s'en résulte. Comment l'État résout-il la question de leur entrée, de leur séjour, de leur statut et de leurs droits en Israël ? En quoi cette migration représente-t-elle une nouvelle donne dans la gestion de migrants non-juifs en Israël et en quoi complique-t-elle les politiques migratoires que l'État est en cours de réviser, contraignant Israël à s'aligner en partie sur le droit international, notamment concernant l'asile ? Enfin, en quoi cette migration est-elle un défi à la nature même d'un État juif en tant que flux clandestin non-juif (donc non voulu) mais aussi un défi éthique justement à un État juif qui se doit, face à son propre passé, d'accueillir des « réfugiés » qui fuient des persécutions religieuses ou ethniques?

\section{I.I - Bref aperçu des migrations non-juives en Israël}

L’État d’Israël est considéré depuis sa création comme un pays d'immigration juive; ainsi, en vertu de la Loi de Retour (1950 et son amendement de 1970), tout individu considéré comme juif (né de mère juive ou converti au judaïsme) peut immigrer et accéder à la citoyenneté israélienne ainsi que tout(e) conjoint(e), enfant ou petit-enfant d'un juif et tout conjoint de l'enfant et du petit-enfant d'un juif. On ne reviendra pas sur les différentes vagues de migrations juives depuis 1948, les dernières étant celle de l'ex-Union soviétique avec plus d'un million d'immigrants entre 1980 et 1990 et celle d’Éthiopie avec près de
70000 immigrants entre 1984 et 2006. Or au début des années 1990 Israël voit de nouveaux flux migratoires sur son territoire: les migrations non-juives de travail, venues remplacer la main-d'œuvre palestinienne après la première Intifada. En effet, le bouclage de la bande de Gaza et des Territoires Occupés ainsi que les demandes en logement pour les nouveaux immigrants russes contraignent le gouvernement israélien à importer une main-d'œuvre étrangère, non-juive et non-palestinienne, de Thaïlande (dans l'agriculture), de Roumanie, de Chine et de Turquie (dans le bâtiment) ou des Philippines et de l'Inde (dans les soins aux personnes âgées) (Berthomière, 2008). À leur pic (en 2002) ces migrants étaient 240000 soit $9 \%$ de la population active du pays dont $60 \%$ sans papiers L'important pourcentage d'irréguliers vient d'une part du système dominant qui lie le migrant de travail à son employeur (autrement dit, s'il quitte son employeur, il devient irrégulier) et de l'autre, du fait que certains ne quittent pas Israël après l'expiration de leur visa de travail. Mais d'autres filières, africaines (Nigéria, Ghana, Éthiopie) et sud-américaines (Colombie, Equateur et Pérou notamment), viennent se greffer sur ces migrations organisées, les migrants entrant, cette fois, comme touristes et trouvant des emplois sur le marché du travail informel (domesticité, restauration) lucratif et en pleine expansion. Pour lutter contre ce phénomène, une police de l'Immigration fut créée en 2002 avec le but explicite d'expulser les irréguliers. Entre 2002 et 2005, 140000 migrants de travail ont quitté le pays (50000 par des reconduites aux frontières et le reste forcés de partir " volontairement ») dont la quasimajorité des Ghanéens et Nigériens; les migrants de travail sont aujourd'hui 250000 dont 150000 seraient irréguliers ${ }^{3}$.

Si au milieu des années 1990, les premiers demandeurs d'asile arrivent comme touristes, pèlerins ou membres de programmes d'échange et de formation professionnelle (Congolais, Ghanéens, Nigériens), la spécificité de ceux d'aujourd'hui reste que la quasi-majorité franchit clandestinement la frontière israélo-égyptienne, dans le désert du Sinaï, à l'aide de passeurs bédouins. Cette traversée comporte évidemment des risques : se faire voler son argent ou ses papiers, être blessé ou abattu par les tirs des Égyptiens, se faire arrêter, être détenu et parfois renvoyé dans son pays d'origine, que ce soit par les autorités égyptiennes ou israéliennes (Anteby-Yemini, 2008). Mais même au prix d'y laisser sa vie, des milliers de migrants entrent ainsi en Israël, notamment depuis l'été 2007. Aujourd'hui on dénombre près de 17000 demandeurs d'asile en Israël, en majorité d’origine africaine; plus de 6500 sont originaires d’Érythrée, 5000 du Soudan (dont une majorité de chrétiens du Sud-Soudan, environ 1500 musulmans du Darfour et une centaine de la région des monts Nuba), près de 1000 de Côte d'Ivoire, 500 du Congo-RDC, et en nombre plus restreint, d’Éthiopie, de Somalie, de Guinée-Conakry, du Togo, et du Tchad entre autres ${ }^{4}$.

2 Un travail de terrain de plusieurs mois en 2007-2008 m'a permis d'effectuer des entretiens avec des demandeurs d'asile africains et des observations participantes dans des abris d'urgence, des cybercafés, des églises et lors de manifestations à Tel-Aviv. Durant cette période, j’étais aussi bénévole une matinée par semaine dans une ONG israélienne (Hotline for Migrant Workers) me permettant de mieux comprendre le traitement de cette population par les institutions israéliennes et les recours au soutien du monde associatif local.

3 Le Bureau central des Statistiques israélien estime à 220000 le nombre de travailleurs étrangers en Israël à la fin 2008 mais la presse avance des chiffres plus élevés cf. R. Eglash « NGOs Slam Steinitz Plan to Deport Illegal Foreign Workers », Jerusalem Post, 13/04/2009.

4 UNHCR-Israel (fin 2008) et le site Refugee Right's Forum (Update April 2009). 
Les motifs de ces mobilités sont multiples: les Érythréens fuient la conscription obligatoire d'une durée illimitée ou des persécutions en tant que membres d'églises évangélistes, alors que les Soudanais fuient des persécutions religieuses et ethniques, voire le génocide au Darfour, tandis que d'autres quittent des conflits généralisés (Congo-RDC), des situations politiques instables (Côte-d'Ivoire) ou recherchent tout simplement une vie meilleure. Si, dans ces nouveaux parcours entre l'Afrique et Israël, le Soudan, le Maroc, l'Algérie, la Lybie et l'Égypte représentent souvent des étapes de transit, les conditions de vie y sont souvent précaires, avec peu de possibilités de travail, du racisme, la menace de détention et d'expulsion et une insécurité accrue - notamment au Caire depuis la dispersion par la police en décembre 2005 d'un sit-in de migrants soudanais contestant leur traitement par le HCR (Haut Commissariat aux Réfugiés des Nations-Unies), qui a fait officiellement 27 victimes (mais plus de 200 selon les réfugiés) ${ }^{5}$. Il est donc plus judicieux de parler ici de « migrants forcés », plutôt que trancher entre demandeurs d'asile ou migrants économiques.

C'est d'ailleurs souvent par hasard que beaucoup choisissent Israël. Certains ne savent rien sur le pays, n’ont jamais entendu parler de Tel-Aviv ni de la situation politique tendue et découvrent par des compatriotes au Caire les opportunités de travail ou les possibilités d'asile en Israël. Seule une minorité décide délibérément de cette destination, à partir du pays d'origine, par le biais de proches ou de connaissances déjà réfugiées en Israël. Il existe enfin des cas plus rares, se rapprochant du trafic d'êtres humains, où des réseaux d'intermédiaires dans les pays d'origine, comme au Congo ou en Guinée-Conakry, organisent le voyage et les migrants paient des sommes importantes (de 1000 \$ à 4000 \$) à l'avance ou après plusieurs mois de travail forcé en Israël. Il y a donc peu de continuité entre cette nouvelle migration forcée et les migrations économiques antérieures - que ce soit dans les réseaux sociaux, les motifs de la migration et les pays d'origine. Cette migration forcée est composée de jeunes hommes issus de classes moyennes, souvent urbaines et éduquées bien qu’on observe également de plus en plus de familles avec des enfants en bas-âge, arrivant parfois à la suite d'un(e) conjoint(e), ainsi qu'une féminisation de ces flux migratoires.

\section{I.2 - Statuts improvisés et chaos bureaucratique}

Si les migrants forcés représentent donc une nouvelle catégorie migratoire en Israël, leur accueil atteste encore d'une politique incertaine, qui oscille entre répression et protection. Ainsi, en vertu de la Loi pour Prévenir l'Infiltration, tout individu qui franchit clandestinement la frontière israélienne peut, pour raisons de sécurité, être détenu pour une période illimitée, comme le sont actuellement près de
1500 migrants forcés. Avec leur afflux durant l'été 2007, le gouvernement mit en place un centre de détention, attenant à la prison de Ktziot, proche de la frontière égyptienne. Mais la détention est loin d'être systématique, faute de places dans les services pénitentiaires, et la majorité des demandeurs d'asile sont libérés sur-le-champ ou bien parviennent aux premières villes israéliennes sans se faire arrêter... Ainsi, plus qu'un instrument de contrôle migratoire, cette politique de détention est avant tout symbolique et les libérations sont fréquentes s'il s'agit d'une demande d'asile fondée ${ }^{6}$. Quant au rapatriement vers l'Égypte ou le pays d'origine il n'est, pour le moment, pas à l'ordre du jour pour la majorité de ces migrants dont la vie serait mise en danger par une expulsion, vers le Soudan ou l'Érythrée notamment ${ }^{7}$.

Ce chaos juridique, politique et administratif, ou ce que S. Willen (2008) nomme la « turbulence gouvernementale », dans laquelle l'État gère ces migrants forcés crée des situations et des statuts inconnus des migrants de travail et bien souvent incohérents et arbitraires. En effet, bien que signataire de la Convention de Genève (1951) sur le Statut des Réfugiés et du Protocole de 1967, Israël ne possède pas de législation nationale sur l'asile. C'est le HCR seul qui traite les demandes et propose à un comité interministériel israélien les dossiers sélectionnés ${ }^{8}$. Deux statuts sont principalement accordés. D’une part, celui de réfugié statutaire, renouvelable tous les deux ans, octroyant un visa de résidence temporaire, un permis de travail et une couverture médicale. Cependant, le taux de reconnaissance est l'un des plus bas au monde (170 individus reconnus réfugiés depuis 1951), en partie parce qu'Israël n'autorise pas l'accès à cette procédure à la plupart des migrants.

D’autre part, une protection temporaire, renouvelable tous les six mois avec permis de résidence et de travail, décernée, prima facie, aux ressortissants de pays victimes de conflits généralisés comme la Côte-d'Ivoire (de 2003 à 2008), le Sierra Leone (entre 1999 et 2005) et le Libéria (de 2003 à 2007). Aujourd'hui, seuls les ressortissants du Congo-RDC reçoivent encore cette protection (depuis 2003), les autres n'en bénéficiant plus, leur pays étant désormais considérés comme "sûrs » : certains ont été rapatriés, d'autres, restés en Israël, sont devenus irréguliers. S’ajoutent à ces "réfugiés humanitaires », les Soudanais et les Érythréens, bénéficiant actuellement de protection collective avec permis de séjour (parfois limités géographiquement), et souvent de travail, pour 1 à 6 mois renouvelable.

Ainsi, ce système de prise en charge mixte de la demande d'asile à la fois par une institution nationale et internationale, le Ministère israélien de l'Intérieur) et et le HCR, coproduit le statut de réfugié (Akoka, 2008) ainsi que de nouvelles catégories - différentes de celles du migrant de travail, régulier ou irrégulier. Elles créent des hiérarchies

5 Voir B. Harrell-Bond (2006).

6 La détention et les conditions de libération n’ont pu être assouplis que grâce aux contestations dans la société civile et aux pétitions d'ONG locales des Droits de l'Homme auprès de la Cour Suprême d'Israël; toutefois, quand il s'agit d'une procédure de détermination du statut de réfugié (RSDP), la détention peut se poursuivre durant toute la durée de l'examen du dossier, à savoir plusieurs mois.

7 Ce principe de non-refoulement n’a pas été respecté dans deux cas récents de « retour à chaud » vers l’Égypte, en août 2007 et en août 2008 où respectivement 48 et 90 demandeurs d'asile subsahariens ont été reconduits immédiatement à la frontière sans possibilité de demander l’asile (Ha'aretz, 29/08/2008).

8 À l’heure où s’achève la rédaction de cet article débutera le $1^{\text {er }}$ juillet 2009 pour tout demandeur d’asile en Israël le traitement intégral de la demande et l'entretien approfondie par le Ministère israélien de l'Intérieur. 
entre statuts (détenu pour « infiltration », demandeur d'asile « en attente », débouté, réfugié, détenteur d'une protection temporaire, sans statut) mais aussi entre nationalités (qui bénéficient ou non de protection); d'où l'émergence d'un trafic de faux-papiers et la circulation de récits standardisés pour se présenter sous une nationalité plutôt qu'une autre mais aussi la menace constante d'arrestation ou d'expulsion pour les demandeurs d'asile devenus irréguliers (en raison de la cessation de leur protection temporaire ou du refus de leur demande d'asile); leur sort est proche de celui de migrants de travail irréguliers. Toutefois, la majorité de migrants forcés obtiennent une forme ou autre de protection en Israël et pour motifs humanitaires, ne peuvent être expulsés.

\section{I.3 - Migration forcée, défi sécuritaire et devoir humanitaire}

L’arrivée de ces migrants forcés depuis moins d'une décennie représente ainsi une nouvelle figure de la mobilité en Israël qui ne se limite plus à la migration purement économique mais fait appel ici à une politique d'asile distincte d'une politique migratoire de travail, tout en soulevant de façon plus urgente la délicate question de la frontière (et de son brouillage) entre demandeurs d'asile et migrants économiques; car ces migrants forcés fuient bien souvent à la fois la pauvreté, une guerre civile et des persécutions. Pourtant, en Israël, l'expression « réfugiés africains » est la plus courante pour les désigner, sans pour autant faire référence à leur statut juridique réel. Nous ne reviendrons pas ici sur la question épistémologique de la construction de la catégorie de " réfugié », qui se veut avant tout juridique ${ }^{10}$, ni sur son usage dans le contexte israélien (Anteby-Yemini, 2009). Disons simplement que ces migrants forcés, qui déposent tous une demande d'asile en Israël pour ne pas être arrêtés ou expulsés, ne sont pas toujours, dans le sens strict du terme, des « réfugiés » (selon la Convention de Genève) de par la diversité des motifs de leur migration.

Par ailleurs, une autre spécificité de ces mobilités est qu'il s'agit d'un flux qui entre sur le territoire israélien clandestinement, transformant ainsi la frontière « sécuritaire » (face à des infiltrations terroristes) entre Israël et l'Égypte en une frontière " migratoire » (face à des migration irrégulières) (Anteby-Yemini, 2008). La menace à la souveraineté de l'État et ses frontières géographiques représentée par cette migration non-juive non-régulée, donne lieu à une politique répressive. Elle est symbolisée par la détention, de ces migrants forcés dont l'entrée ne peut être contrôlée, à la différence des migrants de travail dont l'entrée sur le territoire (par l'aéroport) est régulée par une politique de visas à durée limitée. Cependant, rappelons qu’Israël mène également une politique de répression contre l'immigration irrégulière non-juive de travail et débutera en juillet 2009 une nouvelle campagne d'expulsion de tout étranger sans papiers ou visa valables, sous l'égide de la récente Autorité de l’Immigration.

Dans le même temps, Israël se trouve pour la première fois en présence de populations qui demandent de facto l'asile, en se trouvant déjà sur son territoire. Par le passé, le gouvernement a fait des gestes humanitaires en octroyant des titres de séjour à divers groupes, mais se trouvant tous à l'extérieur de ses frontières (donc des migrants « choisis»), comme en 1977 et 1979 à une centaine de boat people Vietnamiens ou en 2000 aux soldats de l'Armée du SudLiban (ASL) et leurs familles (Ben-Dor et Adout, 2003). Bien que ces mesures restent exceptionnelles, Israël a décidé d'attribuer à 600 demandeurs d'asile du Darfour, le statut de résident temporaire en raison d'une «affinité de souffrance " (Anteby-Yemini, 2009; Willen, 2008). Cependant, toujours en l'absence d'une législation sur l'asile, Israël tente d'articuler une politique à la fois sécuritaire, qui vise à contenir ces flux migratoires en raison de leur nature nonjuive, et humanitaire, qui ne peut ignorer des demandes d'asile de victimes de persécutions ou génocide, compte tenu du poids du passé juif.

À l'heure actuelle, le gouvernement octroie des protections temporaires censées faciliter le raptriement des migrants forcés à la fin de cet asile territorial, permettant donc de les contrôler en les maintenant dans un statut qui peut à tout moment ne plus être renouvelé. De plus, ni les demandeurs d'asile en attente ni ceux munis d'une protection temporaire ne reçoivent d'assistance financière ou d'assurance médicale, les laissant dans des situations très précaires, surtout quand ils sont sans travail ou malades. En refusant ainsi toute prise en charge des demandeurs d'asile et en les tenant à l'écart des droits sociaux et politiques, Israël vise à décourager une installation définitive. Elle les traite comme migrants de transit. Aussi, cette politique paradoxale de contrôle et d'assistance fait émerger une population avec des droits restreints sans possibilité de poursuivre leur migration. C'est pourquoi on peut se demander si, au fond, les « demandeurs d'asile » constituent une nouvelle catégorie migratoire ou s'ils sont traités comme une migration de travail sous un différent nom... Car en octroyant à la plupart des migrants forcés un statut temporaire de non-citoyens, dépourvus de droits sociaux, le gouvernement rejoint les mêmes logiques visant à décourager l'installation de non-juifs que ceux de sa politique d'exclusion envers les migrants économiques, perçus comme une menace aux fondements ethno-nationaux de l'État juif (Rosenhek, 2007). Aussi est-il pertinent de comprendre comment les demandeurs d'asile, repoussés aux marges la société israélienne, parviennent néanmoins à s’y insérer.

\section{2 - Insertion comme migrants étrangers dans la ville}

Examinons en effet comment, les migrants forcés africains en Israël commencent peu à peu à s’installer, travailler et devenir des résidents (réguliers ou irréguliers) des villes israéliennes et quel rôle jouent ici les migrations africaines précédentes.

Les migrants de travail ont certes contribué au caractère cosmopolite de villes comme Tel-Aviv (Berthomière, 2003) et plus largement, à la globalisation de la société israélienne,

9 Sur la hiérarchie entre victimes de persécutions « politiques » et « économiques ». Voir A. Morice \& C. Rodier (2005).

10 Voir à ce propos les travaux entre autres de L. Malkki (1995) ou F. Le Houérou (2004). 
désormais habituée à la présence d’Africains (même si les migrants économiques nigériens ou ghanéens ne comptent plus que quelques centaines, en majorité des femmes seules avec des enfants). De plus, l'arrivée de migrants économiques est accompagnée par la mise en place d'ONG de soutien et de défense de leurs droits. Les nouveaux migrants reproduisent-ils les modes de vie ou d'action et les pratiques spatiales, sociales et politiques des migrants précédents (en particulier ceux du Ghana et du Nigéria)?

\section{I - Exploitation économique et «business » ethnique}

Dans un premier temps, les demandeurs d'asile qui échappent à la détention (soit faute de place dans les prisons soit par ce qu'ils ne se font pas arrêter) tentent tous de regagner Tel-Aviv afin de déposer une demande d'asile au HCR; une fois des documents de protection obtenus, ils peuvent circuler et rechercher un emploi"'. La majorité de ceux sous protection temporaire ainsi que tous les réfugiés statutaires ont le droit de travailler légalement et se concentrent dans quelques secteurs d'activité: la domesticité (travaux de ménage, garde d'enfants), la restauration, l'hôtellerie, le gardiennage et les travaux de déménagement, de peinture, de bâtiment ou de livraison. Certains n'ont pas d'emplois stables et sont embauchés à la journée ou à la semaine, résultant ainsi en une grande flexibilité sur le marché du travail mais aussi en une vulnérabilité qui mène à des cas d'exploitation économique. D’ailleurs, plusieurs ONG parlent de « main-d'œuvre captive et exploitée »... Rappelons, cependant, qu’à la différence des migrants de travail réguliers, aucun lien ne lie le migrant forcé à son employeur et il peut donc négocier ses conditions de travail et changer d'employeur. De plus, les salaires sont nettement supérieurs à d'autres pays de la région et nombreux sont ceux qui envoient des remises dans leur pays d'origine ou en Égypte. Ainsi Ismaïl ${ }^{12}$, un Soudanais, soutient financièrement sa femme restée au Caire et Bernard, un Ivoirien fait vivre douze personnes de sa famille « au pays ».

Par ailleurs, de par le statut légal de nombre de migrants forcés, un commerce ethnique se développe, et de plus en plus d'Érythréens et de Soudanais ouvrent, surtout à TelAviv, des restaurants, des cybercafés, des bars, des boîtes de nuit, des salons de coiffure et des magasins de téléphonies et d'informatique. Leurs boutiques sont souvent ornées de drapeaux israéliens, d’inscriptions en arabe ou en tigrinya sur les vitrines, de photos de Barak Obama, de posters de leur pays et résonnent de musiques érythréennes ou soudanaises... Il existe aussi des « entrepreneurs » comme Louis, un Ivoirien arrivé en Israël il y a six ans, devenu « agent d'emploi » parmi les migrants forcés pour lesquels il trouve du travail dans les réseaux de restaurants israéliens avec qui il collabore. Si c'est en majorité les hommes qui travaillent, les femmes sont également présentes sur le marché, en particulier dans les travaux de nettoyage et les com- merces ethniques, mais aussi dans des petits boulots telle une Soudanaise qui garde des enfants à son domicile ou des Congolaises qui tressent les cheveux ${ }^{13}$. On observe donc une insertion dans les mêmes niches économiques que certains migrants de travail irréguliers mais également la création de commerces ethniques propres à ces nouvelles communautés africaines, qui desservent également une clientèle multiethnique (des Érythréens mangent dans des restaurants soudanais, des Chinois fréquentent un cybercafé érythréen et des Ghanéennes se font coiffer dans un salon de beauté soudanais). Mais ces espaces représentent surtout des lieux de sociabilité qui recréent de véritables enclaves soudanaises et érythréennes au sud de Tel-Aviv, telles les arrière-boutiques de certains cafés où des dizaines d'hommes jouent aux dominos buvant du café et fumant la shisha devant des écrans géants projetant des films soudanais...

\section{2 - Incorporation urbaine et territorialisation}

Il n'existe aucun centre d'accueil ni aucun droit au logement pour demandeur d'asile ou réfugié en Israël. Des abris d'urgence mis en place par une ONG d'anciens demandeurs d'asile africains, l'African Refugees Development Center (ARDC), accueillent des cas humanitaires, comme des femmes seules avec des enfants en bas-âge, et quelques églises, fondées par des migrants africains précédents, hébergent ponctuellement de nouveaux arrivants. Mais le plus souvent, on partage un appartement avec des proches ou des connaissances faites durant le passage vers Israël ou dans le pays même. Ces réseaux sociaux recoupent les communautés nationales et ethniques, comme l'explique Raoul, un Sénégalais qui partage un logement avec un Ivoirien rencontré au centre de détention de Ktziot et avec un Érythréen, qui travaille dans le même restaurant que lui. Des couples mixtes se forment également, souvent entre migrantes philippines et demandeurs d'asile africains. Mais de par la présence de longue date de migrants de travail, notamment à Tel-Aviv, plusieurs ONG procurent une assistance juridique (Hotline for Migrant Workers), une aide concernant le droit du travail (Kav le Oved) ou des soins médicaux gratuits (clinique des Médecins pour les Droits de l'Homme-Israël) et développent désormais des projets ciblés pour les demandeurs d'asile. Par ailleurs, la loi israélienne sur l'éducation obligatoire permet aux enfants de migrants, quel que soit leur statut, d'être scolarisés - à la différence de l'Égypte par exemple (Le Houérou, 2004) - d'où des jardins d'enfants et des écoles du sud Tel-Aviv qui en accueillent déjà depuis deux décennies. Outre ces services éducatifs municipaux, des crèches "pirates » ouvertes par des migrantes de travail (Colombiennes, Ghanéennes) se chargent de la garde d'enfants de celles qui travaillent, témoignant de l'interaction entre les différentes populations étrangères. Quant aux demandeurs d'asile adolescents, certains ont rejoint des internats publics alors que d'autres suivent des cours à Jaffa donnés par le mouvement de jeunesse travailliste et fréquen-

I I Hormis des rapports sur leur traitement par l’État israélien, aucune étude ethnographique sur la vie des demandeurs d’asile en Israël n’a été publiée à ce jour.

12 Tous les prénoms de mes interlocuteurs ont été changés.

13 Parmi les Érythréens réfugiés au Caire, ce sont les femmes qui travaillent (dans la domesticité) et soutiennent financièrement la communauté (Le Houérou , 2004) alors que celles du Darfour réussissent économiquement dans de nouveaux métiers - tatouages au henné ou vente d’araki et de plats soudanais (Le Houérou, 2008). 
tent le club des jeunes mis en place par l'ARDC à Tel-Aviv. De plus, la section des scouts israéliens (Tzofim) de ces quartiers-sud a intégré de nombreux enfants de migrants forcés. Enfin, le Centre d'aide et d'information pour les communautés étrangères (Mesila) de la municipalité de TelAviv organise des activités extras-scolaires pour les enfants de « réfugiés » et des cours de langue (hébreu, anglais) pour les adultes ${ }^{14}$. Comme pour les migrants de travail, ces pratiques d'incorporation locale de la ville vont de pair avec une politique gouvernementale d'exclusion de migrants nonjuifs (Kemp \& Raijman, 2004).

Ainsi, ce sont progressivement les quartiers-sud de TelAviv (Neveh Shanan, Shapira, Florentine, HaTikva), près de la gare routière centrale - où vivent en majorité des migrants de travail ${ }^{15}$ - qui deviennent des espaces de concentration privilégiés pour les migrants forcés, en raison des bas loyers, des opportunités d'emplois, de la proximité d'ONG et de services (commerces ethniques, centres de téléphonie, églises africaines, boutiques de transferts monétaires). De même que dans d'autres contextes urbains tels Istanbul, la localisation des migrants dans la ville répond à une logique de «proximité par rapport aux lieux-ressources, à une logique de réseaux et une logique d’opportunités en matière d’offre immobilière » (Aslan et Pérouse, 2003: 193). Ainsi voit-on apparaître à Tel-Aviv un véritable «quartier africain» (comme à Alger, Naples ou Tripoli) avec des processus de territorialisation et de localisation proches de ceux mis en place par les migrants économiques, devenus de « nouveaux Tel-Aviviens », incorporés dans la ville comme « citoyens urbains » (Kemp et Raijman, 2004). Certains demandeurs d'asile sont également dispersés dans les banlieues de TelAviv, ou à Jérusalem, d'autres travaillent dans les hôtels de la station balnéaire d'Eilat ou dans des collectivités agricoles (moshavim ou kibbutzim) à travers le pays.

Cependant, depuis février 2008 des restrictions dans les permis de travail et de résidence obligent désormais la plupart des migrants forcés à s’installer au nord de Hadera et au sud de Gadera, excluant la région de Tel-Aviv pour y empêcher une « crise humanitaire »; beaucoup déplorent l'absence de structures communautaire ou d'ONG dans les villes périphériques. De plus, en novembre 2008, le maire d'Eilat déclare ne plus pouvoir accueillir 2000 demandeurs d'asile soudanais installés dans cette ville car les services sociaux, médicaux et scolaires y sont débordés, sans subside de l'État pour accommoder cette nouvelle population ${ }^{16}$. De nombreux migrants partent ainsi pour Arad, trouvant un emploi dans les hôtels de la mer Morte. Cependant, si cette dispersion géographique contraste avec les migrants de travail, concentrés à Tel-Aviv, une présence massive à
Arad ou à Eilat suscite également des oppositions dans la population locale ${ }^{17}$. Toutefois, cette politique de contrôle des migrants forcés, par l'interdiction d'installation à Tel-Aviv, vient d'être levée en juillet 2009 par la Cour Suprême.

Ainsi, peut-être à la différence des migrants de travail, l'usage des territoires urbains par les migrants forcés répond à des logiques à la fois de mobilité et de sédentarité car il s'agit de populations qui n'ont plus toujours de « chez-soi » vers lequel retourner et qui ignorent vers où ils iront ensuite. Cette façon « d’habiter le mouvement », pour reprendre une formule d'A. Tarrius, signifie que l'expérience de la déterritorialisation n'est pas toujours la même que celle d'un migrant de travail qui envisage un retour « au pays », d'autant plus que ces migrants forcés ont vécu plusieurs semaines, plusieurs mois, voire plusieurs années dans des lieux de transit (au Soudan, en Égypte, dans les pays du Maghreb, en Lybie ou dans des pays africains), développant des stratégies de survie, souvent comme réfugiés urbains et des compétences et savoir-faire comme transmigrants. Enfin, ces mobilités créent de nouvelles chaînes migratoires sans lien avec les migrations économiques précédentes en inscrivant Israël dans de nouveaux territoires de circulation; l'échange constant d'informations, par téléphone portable ou internet, avec le pays d'origine et l'Égypte ${ }^{18}$, détermine ainsi les décisions de migrer en fonction du statut obtenu, des conditions de travail et des droits acquis en Israël par des proches.

\section{3 - Vie communautaire}

Certes, les nouvelles technologies jouent un rôle considérable dans la construction des réseaux sociaux, que ce soit le téléphone portable, souvent égyptien, que possède pratiquement tout demandeur d'asile (ainsi que ceux détenus près de Tel-Aviv), ou l'internet, largement utilisé dans les cybercafés ou sur les ordinateurs personnels achetés en Israël, permettant aussi de suivre la situation politique dans le pays d’origine. La télévision par satellite joue également une fonction centrale comme source d'informations et de loisirs, telle la chaîne francophone TV5 pour les Congolais ou les chaînes du monde arabe pour les Soudanais. Des groupes de musique (chants congolais, hip-hop sud-soudanais) et de danse (Nuer, Four, Massalit) se sont aussi créés et des concerts de chanteurs africains venus d'Éthiopie ou d'Angleterre par exemple, ont lieu régulièrement, d’où une vie culturelle et sociale active. Enfin des liens existent avec les Israéliens d’origine éthiopienne et nombre d’Érythréens (certains locuteurs d'amharique) mangent dans leurs restaurants, sont employés dans leurs commerces ou achètent des produits dans leurs magasins situés autour de la gare routière de Tel-Aviv.

14 Mesila estime à 5000 personnes la population de demandeurs d’asile africains à Tel-Aviv (rapport 2008).

15 Tel-Aviv regroupe la plus grande communauté de migrants de travail du pays avec 50000 personnes de 80 pays (Schnell, 2007 : 88 ).

16 R. Sinai "Israel to expel 2000 African Refugees who fled to Eilat", Haaretz, 26/11/2008.

17 Ainsi des manifestations ont eu lieu à Arad où se trouvent entre 700 et 1000 "réfugiés africains » (dont deux églises sud-soudanaises) contre leur installation dans la ville et la concurrence qu'ils représentent sur le marché du travail en acceptant des conditions de quasi-esclavage et des salaires dérisoires (B. Moscona-Lerner et M. Grinber « Arad: ville-refuge », Maariv 17/7/2008 en hébreu). De même dans le quartier défavorisé de Shapira, à Tel-Aviv, le comité des résidents lutte contre la concentration de « réfugiés africains » qui alourdit les services sociaux et scolaires de leur quartier (Y. Haï « Dans le quartier Shapira on ne veut pas encore des Africains », Haaretz 7/08/2008 en hébreu). Voir aussi T. Fenster et H. Yacobi (2005) sur les perceptions des migrants étrangers par les résidents israéliens du sud- Tel-Aviv dont une majorité y voit une menace et une dévaluation du quartier et une minorité qui y voit une touche d'exotisme et de cosmopolitisme.

18 Pour effectuer des appels au Soudan à partir d'Israël, certains possèdent une carte SIM d'un pays européen. 
On assiste, de plus, à une politisation croissante chez ces migrants qui s’organisent non seulement en communauté ethnique (telle l'association Darfur Sons Union in Israel) et nationale (comme l'Association des demandeurs d'asile érythréens en Israël), mais aussi en associations panafricaines, telles l'African Refugees Development Center (ARDC) constitué de ressortissants du Soudan, d'Érythrée, d’Éthiopie et du Libéria entre autres; les représentants deviennent quant à eux les interlocuteurs auprès des autorités israéliennes et du HCR. Il existe également un militantisme politique diasporique, comme l'atteste l'ouverture d'une branche du Mouvement pour la Libération du Soudan (MLS) à Tel-Aviv. Or l'émergence d'un leadership communautaire, la politisation de l'identité et la création d'un espace de revendications sont des processus déjà observés chez les migrants de travail africains, philippins et latinoaméricains en Israël (Kemp et Raijman, 2004; Sabar, 2006).

Mais c'est sans doute la fonction centrale de la religion, comme pour les migrations africaines précédentes ${ }^{19}$, qui joue dans la structuration de ces communautés, qu'il s'agisse d'églises catholiques ou protestantes (locales et africaines). De même que parmi les migrants éthiopiens au Caire, pour qui « l'exil a renforcé la spiritualité » (Le Houérou, 2004, p. 101), les églises jouent un rôle de soutien et de pôle culturel dans la vie des migrants forcés en Israël, organisant concerts, activités sociales, repas de fêtes et messes le samedi (le dimanche étant un jour de travail). On assiste non seulement à la superposition de nouvelles et anciennes églises (Érythréens fréquentant les églises de migrants ghanéens; Ivoiriens fréquentant les églises congolaises) mais aussi à une continuité migratoire (telle une église sud-soudanaise fondée au Caire et reconstituée en Israël) ou encore des glissements avec le judaïsme, tels des migrants qui portent d'ostentatoires étoiles de David ou qui ornent leurs églises de drapeaux israéliens et d'étoiles de David aux côtés des croix, en invoquant des prophéties de l'Ancien Testament ${ }^{\mathbf{2 0}}$. C'est aussi la première fois qu'Israël reçoit tant de migrants musulmans (majoritairement du Darfour mais aussi d'autres pays africains); toutefois, les relations avec les Arabes israéliens restent tendues et peu de migrants fréquentent les mosquées locales ${ }^{21}$.

Ainsi, malgré leur précarité, beaucoup de migrants forcés parviennent à s’insérer économiquement (par le monde du travail), linguistiquement (par la scolarisation des enfants dont certains parlent parfaitement l'hébreu et son apprentissage par les adultes) et socialement (par l'usage de services municipaux et non-gouvernementaux) dans la vie quotidienne du pays. Les relations avec la population israélienne se multiplient et on assiste à la fois à des rapports amicaux et hospitaliers et à des rapports d'exploitation ou du racisme (opposition à l'installation de « réfugiés afri- cains » dans certains quartiers) ${ }^{\mathbf{2 2}}$. Simultanément, les migrants forcés s'intègrent aussi dans le tissu social des migrants de travail en Israël, par l'investissement des mêmes quartiers et territoires urbains, parfois des mêmes lieux (bâtiments d'églises), par le recours aux mêmes ONG et aux mêmes services municipaux ou communautaires (jardins d'enfants « pirates ») et par la fréquentation des mêmes commerces, donc par l'usage de structures créées par les migrants précédents et d'espaces chargés de mémoires des migrations passées. En effet, à la suite des expulsions massives depuis 2002, l'intense vie communautaire ghanéenne et nigérienne (bars, clubs de sport, magasins, coiffeurs, tontines, associations culturelles et politiques, plus de 40 églises) s'est désintégrée (Sabar, 2006). Aujourd’hui, ce sont les nouvelles communautés africaines de demandeurs d'asile qui mettent en place leurs propres structures religieuses, économiques, politiques et culturelles, offrant une vie sociale dense, une solidarité ethnique et une organisation associative dynamique qui les transforment en véritables diasporas, liées aux autres communautés soudanaises ou érythréennes exilées dans le monde, tout en transformant Tel-Aviv en « ville-monde » (Berthomière, 2003) désormais lieu de refuge supplémentaire de ces diasporas africaines globales de migrants forcés. Cependant, s'érigent aussi des frontières, entre statut et nationalité, ainsi qu'entre migrants de travail et migrants forcés, en raison d'une visibilité différente et de revendications qui identifient ces derniers et par lesquelles ils s'identifient en tant que « réfugiés », convoquant une rhétorique de « diasporas de victimes ».

\section{3 - Visibilité comme « réfugiés » dans la ville}

Les migrants forcés africains ont en effet recours à des formes d'action, d'organisation politique et de contestation en rupture avec celles des migrants de travail, en mobilisant la société civile israélienne pour soutenir leur cause et en investissant l'espace public de manière inédite. Cette spécificité identitaire les rend plus visibles et plus à même de mériter l'attention et la compassion de la société d'accueil que les migrants économiques. Du coup, ils ont une pratique différente de la ville, ne serait-ce que dans leurs déplacements, accédant à une visibilité à plusieurs niveaux, qui leur donne une nouvelle forme de présence dans l'espace urbain. De plus, en produisant un discours et des revendications qui font usage du passé juif tout en étant liés au registre de l’humanitaire, les représentations et les demandes des migrants forcés les associent à des « réfugiés ", donc une image et un statut différents de ceux d'autres migrants non-juifs en Israël.

19 Cf. Sabar (2006) sur les églises de migrants de travail africains en Israël.

20 Des pratiques similaires d'emprunts au judaïsme se retrouvent chez les migrants de travail des Philippines ( Liebelt, 2008) et d’Amérique latine (Kemp et Raijman, 2003) en Israël.

21 À ce titre, certains Soudanais m’ont parlé de leur craintes de fréquenter les Arabes israéliens, qui leur sont hostiles. De même, on observe peu de contacts entre migrants de travail chrétiens et Arabes chrétiens locaux (Kemp et Raijman, 2003; Sabar, 2006).

22 Ces interactions contrastent avec les relations négatives entretenues avec la population en Égypte en raison de rumeurs (trafic d'organes), d'abus (exploitation, racisme, insultes) et de mythes urbains (les Africains sont porteurs de maladies) à l'exception de la charité musulmane lors des « tables de Ramadan » (Le Houérou, 2004). 


\section{I - Investissement de l'espace public, de la société civile et de l'arène politique}

Si les migrants forcés commencent à investir les quartiers des migrants de travail au sud de Tel-Aviv, ils sont aussi en passe d'en changer le paysage humain avec désormais une dominante africaine, de nouvelles langues (arabe, tigrinya et dialectes soudanais) et de nouvelles religions (islam africain). Ils s’approprient également certains lieux, comme l'aire de jeux, les pelouses et le terrain de basket du parc Lévinski, à proximité de la gare routière, ou les ruelles du quartier Shapira, transformées en terrains de jeux et en espaces de sociabilité. Si ces migrants s’aventurent peu dans le reste de Tel-Aviv, beaucoup se repèrent par rapport aux bureaux des ONG ou du HCR (où l'on me donne souvent rendez-vous); en revanche, ils connaissent tous le marché (Shuk ha-Carmel) et découvrent peu à peu les plages longeant la ville. Les enfants sont de plus en plus nombreux dans les classes, comme à l'école Bialik au sud de Tel-Aviv, mais aussi dans les écoles d'Eilat par exemple où peu d'enfants de migrants africains étaient scolarisés auparavant. Les demandeurs d'asile africains transforment également la spatialité des villes périphériques, qui ont connu peu de migrants (Arad, Sderot, Tibériade, Hadera), y opérant une "globalisation par la marge ", pour reprendre une expression d'A. Bensaâd.

Toutefois, leur nouvelle présence dans l'espace urbain se manifeste avant tout par les formes de contestation que ces migrants forcés utilisent, à savoir les manifestations, les tracts, les marches silencieuses, les sit-in et les pétitions (diffusées à la Knesset, sur internet, dans les universités ou dans la rue). Leurs manifestations devant des lieux stratégiques (le Parlement à Jérusalem, le Ministère de l'Intérieur, de la Défense ou la cinémathèque à Tel-Aviv) accentuent leur visibilité dans l'espace public jusqu'à même investir l'espace virtuel du net, avec le blog d'un réfugié congolais sur le site de la municipalité de TelAviv... D’ailleurs, outre leur large couverture médiatique, les migrants forcés sont dépeints dans la presse locale de Tel-Aviv comme résidents de la ville Ainsi, de par le statut légal de nombreux demandeurs d'asile, leur présence dans l'espace public devient légitime voire légitimée, déployant des pratiques spatiales différentes de celles de migrants économiques, notamment irréguliers, qui préfèrent rester invisibles et ne pas manifester publiquement (Kemp \& Raijman, 2003; Willen, 2007).

Mais c'est surtout au niveau de la société civile israélienne que ces «réfugiés » ont eu le plus d'impact. Tout d'abord, des citoyens se sont spontanément mobilisés pour les accueillir lors des arrivées massives de l'été 2007 dans un élan d'empathie et de solidarité (Anteby-Yemini, 2009); Willen, 2008). Des comités de soutien, comme le
Committee for the Advancement of Refugees from Darfur, ont été créés par des militants israéliens et des personnalités du monde universitaire et, récemment, le Refugee Rights' Forum a été mis en place, constitué de $8 \mathrm{ONG}^{23}$. Parallèlement, depuis 8 ans, une Journée Internationale des Migrants est organisée à Tel-Aviv par des associations pour les droits de l'homme et les deux dernières années ont particulièrement souligné le sort des « réfugiés africains " en donnant la parole à des demandeurs d'asile du Darfour. De plus, un programme sur le droit des réfugiés à l'université de Tel-Aviv s’implique également dans les recours en justice en faveur des demandeurs d'asile tout en essayant de promouvoir une législation sur l'asile en Israël.

Enfin, les migrants forcés sont de plus en plus pris en compte au niveau de l'État, que ce soit par le début de nationalisation de la procédure d'asile (dont un bureau du ministère de l'Intérieur s'occupe désormais d'enregistrer les demandes d'asile et de procurer les visas de séjour et de travail) ou dans les débats à la Knesset, où certains parlementaires se mobilisent contre le refoulement des migrants forcés vers l'Égypte, invoquant le devoir moral d'Israël de protéger des « réfugiés » en tant qu’État juif alors que d'autres s'opposent à les accueillir justement parce qu'il s'agit de migrants non-juifs. Ces discussions nourrissent également la réflexion actuelle pour réviser les lois migratoires en Israël, d'autant plus qu'un nombre croissant de migrants forcés arrivent avec leur famille ou en fondent, par opposition au peu de migrants de travail qui entrent dans le pays avec des enfants ou des conjoints. D’ailleurs, donner naissance apparaît, pour certains, comme la meilleure façon d'obtenir des permis de séjour ${ }^{24}$. Ainsi Gabrielle, une Congolaise, pense qu'avec un enfant « c'est plus facile pour rester en Israël »; Marie du Sud-Soudan déclare « avec un enfant né en Israël, on a plus de chance de rester et même de devenir Israélien »; et Sophie une Ghanéenne affirme que « les Israéliens n'expulsent pas les femmes enceintes ou avec des enfants ». Bien que ces discours ne relèvent que de la rumeur, puisqu'Israël n’a pas de droit du sol, ils se fondent sur un décret exceptionnel et non renouvelable, qui a permis, pour motifs humanitaires, aux enfants de migrants non-juifs de demander une régularisation auprès du Ministère de l'Intérieur ${ }^{25}$. Toutefois, quant aux enfants de migrants forcés nés en Israël, aucune mesure n’est en vigueur à l'heure actuelle - ce qui signifie que ceux qui ne sont pas enregistrés à l'ambassade du pays des parents (par choix ou par absence de représentation diplomatique en Israël) restent apatrides, le ministère israélien de l'Intérieur ne délivrant qu'un extrait de naissance sans droit à la nationalité. Pourtant, des familles africaines donnent souvent des prénoms israéliens aux enfants et encadrent leur photo arborant une étoile de David au cou...

23 Assaf, Physicians for Human Rights-Israel, Hotline for Migrant Workers, Kav le’Oved, Association for Civil Rights in Israel, Amnesty International, African Refugee Development Center et le Programme sur le Droit des réfugiés de la Faculté de Droit de l'université de Tel-Aviv.

24 Notons que cette tendance s’oppose aux stratégies mises en place par les Éthiopiennes au Caire, qui en situation de transit, choisissent de ne pas avoir d'enfants (Le Houérou, 2004: 97).

25 Ce décret de 2005 ne s’applique qu’aux enfants de migrants entrés légalement en Israël, âgés de 6 ans et plus, nés en Israël ou y vivant depuis au moins 5 ans, parlant l'hébreu, scolarisés dans le système israélien et ayant fait une demande avant août 2006. S'ils obtiennent un permis de résident permanent, après leur service militaire, ils recevront la nationalité israélienne et parents et germains auront le statut de résidents temporaires. Environ 650 enfants seraient candidats à cette possibilité de naturalisation (Kemp, 2007). 
Ainsi, les migrants forcés témoignent d'une visibilité accrue dans l'espace public, dans la société civile et dans l'arène politique; mais cet investissement spatial inédit résulte aussi d'une prise de parole qui fait entendre leurs voix comme victimes de persécutions.

\section{2 - Discours de victimes et usage local du passé juif}

Les migrants forcés font appel à leur souffrance et aux persécutions subies tout en établissant un parallèle avec l'histoire juive et le passé de "réfugiés » de certains Israéliens pour invoquer hospitalité et asile en Israël ${ }^{26}$. Ce discours utilisé par les demandeurs d'asile provient aussi d'une rhétorique élaborée par les militants israéliens et les ONG locales que les migrants se réappropient et reprennent à leur compte. À ce titre, la phrase, tirée de la Bible « Tu ne maltraiteras pas l'étranger et tu ne l'opprimeras point; car vous-mêmes avez été étrangers dans le pays d’Égypte » (Exode 22 :20) apparaît déjà dans maintes brochures d'ONG israéliennes des années 1990 qui militent pour le traitement humain des migrants de travail mais est désormais reprise pour exiger l'accueil de « réfugiés » africains en Israël. Ainsi, des migrants congolais, lors d'une manifestation (déc. 2007), au centre de Tel-Aviv, pour attirer l'attention sur leur sort, exposent des panneaux avec des photos et des textes qui montrent les horreurs au Congo-RDC (viols, meurtres collectifs, fosses communes, enfants-soldats) et des pancartes « Nous sommes tous des réfugiés », " l'asile maintenant ». L'usage même du terme " réfugiés », par les migrants forcés et les associations qui les soutiennent, tente également de lier cette désignation au passé juif et particulièrement à celui de citoyens israéliens arrivés comme réfugiés après la Seconde Guerre Mondiale (notons ici qu’à aucun moment il n’y a de références aux Palestiniens). Cette obligation morale de donner refuge aux demandeurs d'asile africains est parfaitement illustrée par le texte d'un clip de l'Association pour les Droits Civils en Israël ${ }^{27}$ : « L’asile pour les réfugiés. C’est juste (...) c’est notre devoir ». Quant aux Soudanais, en particulier ceux originaires du Darfour, ils n’hésitent pas à comparer l'horreur du génocide au Soudan à celle de la Shoah en Europe (Anteby-Yemini, 2009; Willen, 2008), revêtant par exemple des t-shirts avec «Darfour-génocide-Shoah » inscrit en anglais, hébreu et arabe et créant des associations pour faire connaître leur sort, telles le collectif Darfur Sons Union in Israel.

Parallèlement, un discours qui fait usage du passé d'exil du peuple juif tente également de justifier la migration et le refuge des migrants en Terre Promise. C'est en particulier par le christianisme et les épisodes bibliques (sortie d’Égypte) qu'Israël est présenté comme un pays saint ${ }^{\mathbf{2 8}}$. À ce titre, des migrants ivoiriens qui protestaient devant le Ministère de l’Intérieur à Tel-Aviv (déc. 2008) contre la cessation de leur protection temporaire, brandissaient des panneaux avec les textes suivants: «Israël et l'hospitalité comme mode de vie », « On aime Israël, béni soit l’État d’Israël ». Lors d'une autre manifestation, des Sud-Soudanais scandaient des slogans chrétiens prophétiques et arboraient des pancartes qui disaient - « Les Sud-Soudanais ne sont pas des touristes en Israël », « Mubarak est le boucher des Soudanais », justifiant leur fuite d'Égypte et leur refuge en Israël. C'est ainsi que les migrants forcés parviennent à articuler un discours et des revendications fondées sur la souffrance commune de persécutions, l'expérience partagée de « victimes de génocide » et la recherche similaire d'une terre d'asile pour les Israéliens d'hier et les demandeurs d'asile africains d'aujourd'hui. Ce rapprochement permet de réunir les migrants forcés et la population d'accueil sous le même statut de "diaspora de victimes ", pour emprunter les classifications de R. Cohen (1997), et donc de donner à cette identification une signification toute particulière dans le contexte local israélien, qu'elle n’a jamais eu en Égypte ou ailleurs. Simultanément, ces migrants font aussi usage d'un discours plus global sur l’asile.

\section{3 - Rhétorique de l'humanitaire et droit global des réfugiés}

En effet, des migrants forcés africains se sont insérés dans des structures déjà existantes, comme l'Union des Travailleurs Africains, fondée par les migrants de travail nigériens et ghanéens pour défendre leurs droits et promouvoir la cause des irréguliers dans la sphère publique et politique, en convoquant un langage global des droits de l'homme relayé par les ONG locales et leur lobbying au Parlement (Kemp et Raijman, 2004 ; Sabar, 2006). Mais surtout, ils créent leurs propres organisations aux revendications spécifiques, telles l'African Refugee Development Center, créée en 2004 pour défendre, promouvoir et revendiquer leurs droits en tant que « réfugiés » et s’insérer dans le contexte global d'un discours humanitaire sur l'asile.

Il existe, cependant, différentes revendications selon les nationalités et les statuts en Israël. Tout d'abord, la demande d'une protection de base (l'asile territorial) se retrouve chez nombre de migrants forcés qui désirent tout simplement trouver refuge en Israël, tels les Ivoiriens qui manifestent en décembre 2008 (avant expiration de leur protection temporaire, le HCR ayant déclaré la Côte d'Ivoire pays « sûr ») et distribuent des tracts qui titrent « Laissez-nous rester encore en Israël après les élections en Côte d'Ivoire », tout en rappelant l'injonction biblique d'indulgence envers l'étranger. L'un des représentants du Comité des réfugiés ivoiriens d'Israël m'explique: "c'est comme si ta maison brûle et tu es hébergé chez ton voisin; tu ne vas pas rentrer tant qu'il y a encore le feu! ».

26 Liebelt (2008) observe que les travailleurs domestiques philippins utilisent également un langage de victimes et d'identification avec la souffrance juive pour négocier des droits sociaux et politiques en Israël, en comparant leur destin à l'histoire des juifs en exil, au point de scander le slogan « nous sommes les juifs d'aujourd'hui » lors de manifestations contre l'expulsion des irréguliers.

27 http://www.acri.org.il/camp/clips/refugees2008eng.html consulté le 10/11/2008.

28 Notons que les églises évangélistes latino-américaines en Israël ont invoqué le christianisme sioniste des migrants pour légitimer leur présence et revendiquer leur inclusion en Israël (Kemp et Raijman, 2003) de même que les églises des migrants de travail africains ( Sabar, 2006), sans pour autant obtenir droit au séjour. 
D’autres, qui bénéficient de protection à titre humanitaire (Érythréens, Soudanais) demandent quant à eux l'accès à la procédure de détermination de statut de réfugié (RSDP), qu'Israël leur interdit, ainsi que l'octroi de droits sociaux comme résidents de facto dans le pays. En effet, n’ayant pas accès à cette procédure, ils ne peuvent être reconnus comme réfugiés statutaires selon la Convention de Genève et du coup n’ont pas les droits afférents à ce statut. Les Sud-Soudanais, par exemple, essaient de faire valoir leurs droits comme réfugiés (parfois reconnus en Égypte) ${ }^{29}$ et l'Association des demandeurs d'asile érythréens en Israël a organisé en décembre 2008 une marche fort impressionnante au centre de Tel-Aviv (environ 500 personnes portant des masques blancs) pour demander l'accès à cette procédure, l'arrêt des refoulements vers l'Égypte, le droit de s'installer à Eilat et la libération des détenus, tout en soulignant leur désir de s’intégrer en Israël. Cette protestation contre la politique israélienne à leur égard faisait aussi appel à la population israélienne pour diffuser leur message « non seulement au niveau local mais aussi au niveau global ». De manière générale, les migrants forcés revendiquent ainsi le droit de demeurer sur le territoire israélien et de recevoir une protection de base mais aussi (pour ceux qui détiennent ces protections) d'obtenir des droits sociaux et médicaux, le droit de travailler et d'étudier (pour les adultes) sans restrictions sur leur mobilité, en invoquant un discours humanitaire global fort différent des revendications des migrants de travail. Le discours des migrants forcés s'articule donc d'une part autour d'une notion d'asile humanitaire universelle, comme droit de l'homme fondamental, et d'autre part autour de la revendication de droits sociaux et d'accès à la demande d'asile statutaire, comme droit international pour tout réfugié. Ces mobilisations politiques se retrouvent dans différents pays où vivent les migrants forcés subsahariens (et reproduisent ici les revendications déjà faites au sit-in du Caire, qui s'est terminé tragiquement) d'où une dimension également transnationale à ces contestations.

On observe ainsi chez les migrants forcés en Israël des revendications locales d'un côté, liées à une rhétorique en tant que victimes de persécutions ou de génocide, spécifiquement tournée vers le public israélien, et globales de l'autre, qui rejoignent un discours transnational sur le droit des réfugiés. Contrairement aux migrants de travail, il s'agit donc d'un nouveau langage du «demandeur d'asile et du réfugié » qui émerge dans la société israélienne, avec l'aide d’ONG locales, faisant entrer, bon gré malgré, Israël dans ce défi global face à l'asile. Enfin, les demandes des migrants forcés et les mobilisations dans la société civile semblent leur octroyer une plus grande « légitimité » face aux migrants économiques pour qui la population d'accueil s'est peu mobilisée et qui suscitent bien moins de compassion, malgré leur exploitation économique et leurs conditions de vie déplorables largement relatées par les média et les associations des droits de l'homme qui militent en leur faveur depuis les années 1990.

\section{Conclusion}

L’arrivée massive de demandeurs d'asile africains en Israël reconfigure certes le paysage migratoire israélien. Nous avons tenté de montrer que les nouvelles migrations forcées vers Israël, tout en étant en transit, reproduisent des modèles de territorialisation, d'insertion économique et de structuration communautaire en continuité avec les migrants de travail des années précédentes. Quant à l'État, il mène une politique semblable à la gestion des autres flux migratoires non-juifs, à savoir décourager l'installation en Israël et avoir recours à la détention ou l'expulsion de sans-papiers; mais ici s'ajoute aussi une logique humanitaire face à des « réfugiés » et même des « rescapés ».

Ainsi s'observe une rupture avec les migrations antérieures car les migrants forcés développent des projets migratoires différents de ceux de migrants économiques - surtout quand un retour est impossible - en recherchant avant tout un lieu de refuge et non simplement une opportunité économique; la plupart n'aspirent pas à gagner de l'argent pour acheter un commerce ou construire une maison dans leur pays d'origine mais plutôt à survivre en exil (et obtenir une protection quand leur vie est en danger), témoignant des stratégies divergentes entre mobilités volontaires et forcées. De plus, la spécificité des demandeurs d'asile en Israël, qui font appel à un discours local comme victimes de persécutions et à un discours global comme réfugiés, représente un défi à la fois éthique et politique pour Israël, l'obligeant à se tourner vers son propre passé comme pays de réfugiés mais aussi à s'engager dans une politique d'asile qu'il a toujours refusé en tant qu'État juif. Cette tension entre l'humanitaire et le sécuritaire conduit donc à des questions distinctes de celles posées par la migration de travail. Par conséquent, les demandeurs d'asile africains, bien qu’aux marges de la société israélienne, parviennent à susciter un débat dans l'espace public et dans la sphère politique, à mobiliser la société civile en leur faveur et à donner une forte visibilité à leurs contestations pour exiger des droits, sans doute bien plus que d'autres étrangers en Israël.

C’est pourquoi, malgré leur statut précaire et l'absence de droits sociaux, les demandeurs d'asile en Israël s'intègrent sans doute mieux que certains migrants de travail et même certains Israéliens d'origine éthiopienne. Cette adaptation peut s'expliquer par le niveau d'éducation souvent élevé des migrants forcés, leurs compétences acquises au cours d'expériences précédentes d'exil et le soutien d’ONG. Aussi, beaucoup adoptent un mode de vie de plus en plus israélien, apprennent l'hébreu, ouvrent des commerces et certains voient même leur avenir en Israël sans savoir s'ils pourront y rester. Tel-Aviv est ainsi en passe de devenir un nouvel espace de transit pour ces mobilités africaines de la même façon qu'Istanbul ou le Caire le sont pour l'Europe. Mais Israël pourrait aussi devenir un pays d'asile pour ceux qui ne pourront rentrer dans leur pays, d'autant plus qu'en raison de la fermeture des frontières de l'Europe et de « l'externalisation de l'asile », ils ne peuvent quitter le territoire israélien sans risquer de ne plus y être réadmis et, à l'heure actuelle, ne poursuivent pas leur voyage. Il est, à ce titre, pertinent 29 En Égypte, la reconnaissance par le gouvernement du statut de réfugié permet de vivre légalement dans le pays, recevoir des soins médicaux dans
certains dispensaires et parfois d’être réinstallés dans un pays occidental (Le Houérou, 2004). 
de ré-envisager la notion de mobilité quand des demandeurs d'asile sont confrontés à une «sédentarité forcée» qui d'une certaine manière les retient dans un pays qui n'accueille pas, officiellement, de réfugiés non-juifs.

Ainsi, Israël rejoint pour la première fois les pays qui deviennent involontairement de nouvelles terres d'accueil et s’implique donc dans un "régime humanitaire » qui exige une législation sur l'asile - inexistante en tant que telle. Cette situation pose aussi des questions que connaissent déjà les pays européens vis-à-vis des demandeurs d’asile (concernant leur intégration et leurs droits sociaux et politiques, le statut juridique de la seconde génération...) et qu’Israël découvre à son tour. L'accès à la nationalité est quasi-impossible pour ces migrants non-juifs, qui au mieux conserveront un statut temporaire, mais soulève néanmoins le débat sur les frontières de l'appartenance à la société israélienne, qui incluent par certains processus les "réfugiés africains » (légitimité de leur présence par devoir humanitaire) et les excluent par d'autres (absence de droits pleins), testant ainsi «les limites de la citoyenneté » (Soysal), de manière semblable mais aussi distincte des migrants de travail.
Enfin ces circulations exigent de considérer la migration de transit et la migration forcée comme de nouveaux paradigmes d'étude de la migration non-juive en Israël, limitée jusqu'ici à la migration de travail. Plus vastement, cet article a voulu présenter l'émergence de nouvelles filières migratoire sur l'axe Afrique/Israël lorsque d'autres destinations ne sont plus accessibles ou sont moins favorables. Cette dimension supplémentaire dans les parcours migratoires de pays africains vers la Méditerranée témoigne ainsi de la globalisation du phénomène des demandeurs d'asile sub-sahariens dans des régions du pourtour méditerranéen (Libye, Maghreb, Liban) qui n'en avaient pas toujours accueillis auparavant. C'est pourquoi, ces mobilités donnent lieu à l'émergence de nouvelles diasporas africaines en Israël et de nouveaux sites de reproduction de la culture soudanaise ou érythréenne, par exemple, dans l'espace méditerranéen mais qui créent aussi de nouveaux réseaux transnationaux qui lient désormais TelAviv avec le Caire, Tripoli, Asmara, Khartoum ou même les villages du Darfour.

\section{Bibliographie}

AKOKA K., (2008), La demande d’asile en Israël, Cambrezy L. et al. (éds), L'asile au Sud, La Dispute, Paris, p. $43-67$.

Anteby-Yemini L., (2008), Migrations africaines et nouveaux enjeux de la frontière israélo-égyptienne, Cultures \& Conflits, 72, p. 75-97, [http://www.conflits.org/index13683.html].

ANTEBY-YEMINI L., (2009), Les ‘réfugiés soudanais’ en Israël: discours, représentations, mobilisations, Maghreb-Machrek, 199, p. 71-83.

ASLAN M. et PÉROUSE J.-F., (2003), Istanbul : le comptoir, le hub, le sas et l'impasse. Fonctions dans le système migratoire international, Revue Européenne des Migrations Internationales, 19-3, p. 173-204.

BEN-DOR A. et ADOUT R., (2003), Israel-A Safe Haven? Problems in the treatment offered by the State of Israel to Refugees and Asylum Seekers, Report and Position Paper, Buchmann Faculty of Law, Tel Aviv University and Physicians for Human Rights, 93 p.

BERTHOMIÈRE W., (2003), L'émergence d'une Tel-Aviv cosmopolite ou les effets d'un fin mélange entre reconfigurations sociopolitiques internes et externes, Cahiers de la Méditerranée, 67, p. 299-312.

BERTHOMIÈRE W., (2008), Les travailleurs étrangers, figures de la « mondialisation d'Israël », Dieckhoff A. (éd.), L'État d'Israël, CERI-Fayard, Paris, p. 205-216.

COHEN R., (1997), Global diasporas: an introduction, University of Washington Press, Seattle, 228 p.

FENSTER T. et YACOBI H., (2005), Whose City is it? On urban Planning and Local Knowledge in Globalizing Tel AvivJaffa, Planning, Theory and Practice, 6-2, p. 191-211.

HARRELl-BOND B., (interrogée par Le Houérou F.), (2006), Le drame de la place Mustapha Mahmoud au Caire, [http://terra.rezo.net/article553.html] consulté le 17/01/07.

KEMP A., (2007), Managing Migration, Reprioritizing National Citizenship: Undocumented Labor Migrants’ Children and Policy Reforms in Israel, Theoretical Inquiries in Law, 8-2, p. 663-691.

KEMP A. et RAIJMAN R., (2003), Christian Zionists in the Holy Land: Evangelical Churches, Labor Migrants, and the Jewish State, Identities, 10, p. 295-318. 
- (2004), “Tel Aviv Is Not Foreign to You”: Urban Incorporation Policy of Labor Migrants in Israel, International Migration Review, 38-1, p. 26-51.

LE HOUÉROU F., (2004) Migrants forcés éthiopiens et érythréens en Égypte et au Soudan: passage d'un monde à l'autre, L'Harmattan, Paris, 202 p.

- (2008) Poussières d’instants: la construction de soi et l'invention de nouveaux métiers par les réfugiées du Darfour au Caire, Le Mouvement Social, 225, p.81-97.

LIEBELT C. (2008), "We are the Jews of today": Filipino domestic workers in Israel and the language of diaspora, Hagar, 8-1, pages 63-81.

MALKKI L., (1995), Refugees and Exile: from 'refugee studies' to the national order of things, Annual Review of Anthropology, 24, p. 495-523.

MORICE A. et RODIER C., (2005), Classer-trier migrants et réfugiés: des distinctions qui font mal, Hommes et libertés Revue de la Ligue des Droits de l'Homme, 129, p. 58-61.

ROSENHEK Z., (2007), Challenging Exclusionary Migration Regimes: labor migration in Israel in comparative perspective, Willen S. (ed.), Transnational Migration to Israel in Global Comparative Context, Lexington Books, Lanham, p. $217-232$.

SABAR G., (2006), Une migration inachevée? Réflexions sur la communauté africaine de travailleurs migrants en Israël, à la veille de sa disparition, Les Cahiers des Anneaux de la Mémoire, 9, p. 43-67.

SCHNELL I., (2007), Migrant Workers’ Segregation and Adaptation to the Ethnic City: the case of Tel Aviv, Willen S. (ed.), Transnational Migration to Israel in Global Comparative Context, Lexington Books, Lanham, p. 87-99.

SOYSAL Yasemin, (1994), Limits of citizenship, The University of Chicago Press, Chicago, 244 p.

WILLEN S., (2007), "Flesh of Our Flesh": Undocumented Migrant Workers' Search for Meaning in the Wake of a Suicide Bombing, Willen S. (ed.), Transnational Migration to Israel in Global Comparative Context, Lexington Books, Lanham, p. 159-184.

- (2008), L’hyperpolitique du « Plus jamais ça ! » : demandeurs d'asile soudanais, turbulence gouvernementale et politiques de contrôle des réfugiés en Israël, Cultures \& Conflits, 71-3, [http://www.conflits.org/index16833.html]. 